\title{
Marked reductions in rates of vancomycin- resistant enterococci (VRE) colonization \& disease associated with introduction of a routine hospital-wide bleach cleaning program
}

\author{
ML Grayson ${ }^{1,2}$, AA Mahony ${ }^{1 *}$, EA Grabsch ${ }^{1}$, DR Cameron ${ }^{1}$, RD Martin ${ }^{1}$, M Heland $^{3}$, M Petty $^{3}$, S Xie $^{3}$ \\ From International Conference on Prevention \& Infection Control (ICPIC 2011) \\ Geneva, Switzerland. 29 June - 2 July 2011
}

\section{Introduction / objectives}

To reduce rates of VRE colonization/disease, we introduced a multimodal hospital-wide bleach-based cleaning program $(\mathrm{BBCP})$ that included a new product (sodium hypochlorite $1000 \mathrm{ppm}+$ detergent), new standardised (routine and detailed) cleaning practices \& modified glove/gown protocols to rely on alcohol-based handrub \& sleeveless aprons. Rates of VRE pre- \& post-BBCP were compared.

\section{Methods}

Patients in 4 high-risk wards (liver transplant, renal, ICU, hem/oncology) were screened on admission \& weekly for rectal VRE colonization, \& rates were compared pre-BBCP (Period A [6 mo] - Feb-July 2009) vs post-BBCP (Period B1 \& B2 - Feb-July \& Aug-Jan 2010/11). Rates of VRE bacteremia (per 100 patients blood cultured [100PBC]) \& of urinary tract infection [UTI] were compared - Period A vs B1 \& B2.

\section{Results}

A 37\% reduction in newly recognised VRE colonizations was observed post-BBCP (208/1948 patients screened [Period A] vs 181/2129 [Period B1] vs 143/2141 [Period $\mathrm{B} 2], p<0.0001)$, despite an increase in screening compliance $(68.1 \%$ vs $74.6 \%$ vs $71.9 \%, p=0.061)$ and a stable rate of on-admission VRE colonization (38/1461 [2.6\%] vs 44/1795 [2.5\%] vs 38/1840 [2.1\%], $p=0.34$ ). VRE bacteremia declined from $0.48 / 100 \mathrm{PBC}(14 / 2935)$ pre-BBCP to $0.08 / 100 \mathrm{PBC}(5 / 6194)$ during the 12 mo post-BBCP $(p=0.0002)$, with a reduction in UTI cases (24 [A] vs 19 [B1] vs 17 [B2]).

\section{Conclusion}

The BBCP was associated with a significant reduction in rates of both new VRE colonizations (37\% decrease) \& VRE disease. This approach potentially represents a new paradigm in the management of VRE.

\section{Disclosure of interest}

None declared.

\section{Author details}

${ }^{1}$ Infectious Diseases, Austin Health, Melbourne, Australia. ${ }^{2}$ Medicine,

University of Melbourne, Australia. ${ }^{3}$ Austin Health, Melbourne, Australia.

Published: 29 June 2011

doi:10.1186/1753-6561-5-S6-P24

Cite this article as: Grayson et al:: Marked reductions in rates of vancomycin-resistant enterococci (VRE) colonization \& disease

associated with introduction of a routine hospital-wide bleach cleaning program. BMC Proceedings 2011 5(Suppl 6):P24.

Infectious Diseases, Austin Health, Melbourne, Australia

Full list of author information is available at the end of the article

(c) 2011 Grayson et al; licensee BioMed Central Ltd. This is an open access article distributed under the terms of the Creative Commons 